\title{
Euler Evolution for Singular Initial Data and Vortex Theory: A Global Solution
}

\author{
Carlo Marchioro * \\ Dipartimento di Matematica. Università di Roma I "La Sapienza." Piazrale A Moro 2. \\ I-(0)185 Roma. Italy
}

\begin{abstract}
We study the evolution of a two-dimensional, incompressible, ideal fluid in a case in which the vorticity is concentrated in small disjoint regions and we prove, globally in time, its connection with the vortex model.
\end{abstract}

\section{Introduction}

In this paper we want to study some properties of the behaviour of a non-viscous, incompressible fluid in two dimensions. In particular, we consider the case in which the vort city is initially concentrated in $N$ small disjoint blobs. We follow their evolution during the time and we prove that, in the limit of vanishing regions, their centers of vorticity move as a system of point vortex ([1], for a review see [2]). This result is global in time and it is obtained for vortices of the same sign.

Until now the convergence local in time for vortices of every sign was known [3], global in time for one vortex in a bounded domain [4] and two vortices of different sign in a bounded region [5]. The last two results are very particular and cannot be generalized to a more complicated case. The present paper is a nontrivial generalization of the method used in [3].

The Euler equations in $R^{2}$ for the vorticity are:

$$
\begin{gathered}
\partial_{t} \omega(\mathbf{x}, t)+(\mathbf{u} \cdot \nabla) \omega(\mathbf{x}, t)=0, \\
\nabla \cdot \mathbf{u}=0, \\
\omega=\operatorname{curl} \mathbf{u}=\left(\partial_{x_{1}} u_{2}-\partial_{x_{2}} u_{1}\right), \\
\mathbf{x}=\left(x_{1}, x_{2}\right) \in R_{2}, \quad \omega(\mathbf{x}, 0)=\omega_{0}(\mathbf{x}) .
\end{gathered}
$$

\footnotetext{
* Research partially supported by CNR and Ministero della Pubblica Istruzione
} 
Here $\mathbf{u}=\left(u_{1}, u_{2}\right)$ denotes the velocity field. If the velocity decays at infinity, we can reconstruct the velocity field by means of $\omega$ :

$$
\begin{gathered}
\mathbf{u}(\mathbf{x}, t) \int \mathbf{K}(\mathbf{x}-\mathbf{y}) \omega(\mathbf{y}, t) d \mathbf{y}, \\
\mathbf{K}=\nabla^{\perp} g, \\
\nabla^{\perp}=\left(\partial_{x_{2}},-\partial_{x_{1}}\right), \\
g(\mathbf{r})=-(2 \pi)^{-1} \ln |\mathbf{r}| .
\end{gathered}
$$

As is well known, Eq. (1.1) means that the vorticity is constant along the particle paths that are the characteristic of the Euler system. Therefore,

$$
\omega(\mathbf{x}, t)=\omega\left(\mathbf{x}\left(\mathbf{x}_{0},-t\right), 0\right),
$$

where the trajectory $\mathbf{x}\left(\mathbf{x}_{0}, t\right)$ of a particle of the fluid initially in $\mathbf{x}_{0}$ satisfies:

$$
\begin{gathered}
\dot{\mathbf{x}}\left(\mathbf{x}_{0}, t\right)=\mathbf{u}(\mathbf{x}, t), \\
\mathbf{u}(\mathbf{x}, t)=\int \mathbf{K}(\mathbf{x}-\mathbf{y}) \omega(\mathbf{y}, t) d \mathbf{y}, \quad \mathbf{x}(0)=\mathbf{x}_{0} .
\end{gathered}
$$

We consider $N$ blobs of vorticity supported in $N$ disjoint open regions $\left\{\Lambda_{i, \varepsilon}(0)\right\}$, $i=1 \ldots N$, such that:

$$
\begin{array}{cc}
\operatorname{meas} \Lambda_{i, \varepsilon}=a_{i} \varepsilon^{2}, & a_{i}>0, \\
\Lambda_{i, \varepsilon} \subset \Sigma\left(\mathbf{z}_{i} \mid \varepsilon\left(a_{i}\right)^{1 / 2}\right), & \mathbf{z}_{i} \in R^{2},
\end{array}
$$

where $\Sigma(\mathbf{z} \mid r)$ is a circle of center $\mathbf{z}$ and radius $r$,

$$
\begin{gathered}
\omega_{i, \varepsilon}(\mathbf{x}, 0)=\varepsilon^{-2} \chi\left(\mathbf{x} \mid \Lambda_{i, \varepsilon}(0)\right), \\
\omega_{\varepsilon}(\mathbf{x}, 0)=\sum_{i=1}^{N} \omega_{i, \varepsilon}(\mathbf{x}, 0),
\end{gathered}
$$

where $\chi(\mathbf{x} \mid \Lambda)$ is the characteristic function of $\Lambda$.

Each blob $\Lambda_{i, \varepsilon}(0)$ evolves via the Euler equation in $\Lambda_{i, \varepsilon}(t)$. Because of a divergence-free condition, the evolution is Lebesgue measure preserving. In particular,

$$
\operatorname{meas} \Lambda_{i, \varepsilon}(t)=\operatorname{meas} \Lambda_{i, \varepsilon}(0) .
$$

Moreover, we note that there is a unique weak solution of the Euler equation with initial datum $\omega_{\varepsilon}$, that we denote as $\omega_{\varepsilon}(\mathbf{x}, t)$.

We define the center of vorticity of the evolved region:

$$
\mathbf{M}_{i, \varepsilon}(t)=a_{i}^{-1} \int d \mathbf{x} \mathbf{x} \omega_{i, \varepsilon}(\mathbf{x}, t)=a_{i}^{-1} \int d \mathbf{x} \mathbf{x}_{\varepsilon}(\mathbf{x}, t) \omega_{i, \varepsilon}(\mathbf{x}, 0) .
$$

We want now to compare this evolution with the vortex model.

We consider $N$ point vortices $\mathbf{z}_{i}$ of intensity $a_{i}>0$. The equations of motion are

$$
d \mathbf{z}_{i}(t) / d t=\sum_{j=1, j \neq i}^{N} a_{j} \mathbf{K}\left(\mathbf{z}_{i}(t)-\mathbf{z}_{j}(t)\right), \quad \mathbf{z} \in R^{2}, \quad \mathbf{z}_{i}(0)=\mathbf{z}_{i},
$$


which admit the integrals of motion

$$
\begin{gathered}
H=-(4 \pi)^{-1} \sum_{i, j=1, i \neq j}^{N} a_{i} a_{j} \ln \left(\left|\mathbf{z}_{i}(t)-\mathbf{z}_{j}(t)\right|\right) \quad \text { (Energy), } \\
\mathbf{M}=\left(\sum_{i=1}^{N} a_{i} \mathbf{z}_{i}\right) /\left(\sum_{i=1}^{N} a_{i}\right) \quad \text { (Center of vorticity), } \\
I=\sum_{i=1}^{N} a_{i}|\mathbf{z}|^{2} \quad \text { (Moment of inertia) }
\end{gathered}
$$

It is immediate to note that $H$ and $I$ prevent the collapses. In the sequel we denote by $R_{0}$ the minimal distance between the vortices.

We state now the main result.

Theorem 1.1. For any fixed $t$,

$$
\lim _{\varepsilon \rightarrow 0} \mathbf{M}_{i, \varepsilon}(t)=\mathbf{z}_{i}(t) .
$$

\section{Preof of the Theorem 1.1}

Let us explain the main difference between the present proof and the local one. In [3] we introduced the moment of inertia of each blob and we proved that it remains bounded by $\varepsilon^{2}$ (and so the blob concentrated) until the vorticity is contained in a finite circle around each vortex. This can be proved only for a small time. Here we introduce a smooth version of the moment of inertia and of the total vorticity and we evaluate their change in time. We prove that the filaments going away (if they exist) give a small contribution and this is enough for the proof. Remains open the problem to understand whether these filaments are actually contained in a bounded region possibly vanishing when $\varepsilon \rightarrow 0$. In this direction a partial result is given in Sect. 3 .

Let $\varepsilon<(1 / 20) R_{0}$. We introduce a smooth function $W(r), r \geqq 0$ such that

$$
W(r)= \begin{cases}1 & \text { if } r \leqq R_{1}, \\ 0 & \text { if } r \geqq R_{2}, \\ a & \text { decreasing smooth function otherwise, }\end{cases}
$$

where

$$
W\left(R_{3}\right)=1 / 2 \text {, }
$$

$$
\begin{gathered}
(1 / 10) R_{0}<R_{1}<R_{3}<R_{2}<(1 / 4) R_{0} \\
\left|W\left(\left|\mathbf{x}_{1}\right|\right)-W\left(\left|\mathbf{x}_{2}\right|\right)\right| \leqq C_{1}\left|\mathbf{x}_{1}-\mathbf{x}_{2}\right| \\
\left|\nabla W\left(\left|\mathbf{x}_{1}\right|\right)-\nabla W\left(\left|\mathbf{x}_{2}\right|\right)\right| \leqq C_{2}\left|\mathbf{x}_{1}-\mathbf{x}_{2}\right| .
\end{gathered}
$$

(From now on $C_{i}$ means a constant independent of $\varepsilon$.)

We want to study the quantity

$$
\begin{aligned}
I_{w}(t) & =\sum_{i=1}^{N} \int d \mathbf{x}\left[\mathbf{x}-\mathbf{z}_{i}(t)\right]^{2} W\left(\left|\mathbf{x}-\mathbf{z}_{i}(t)\right|\right) \omega_{\varepsilon}(\mathbf{x}, t) \\
& =\sum_{i=1}^{N} \varepsilon^{-2} \sum_{j=1}^{N} \int_{\Lambda_{j, \varepsilon}(0)} d \mathbf{x}\left[\mathbf{x}(\mathbf{x}, t)-\mathbf{z}_{i}(t)\right]^{2} W\left(\left|\mathbf{x} \varepsilon(\mathbf{x}, t)-\mathbf{z}_{i}(t)\right|\right)
\end{aligned}
$$


and we prove that this quantity vanishes as $\varepsilon^{2}$ when $\varepsilon \rightarrow 0$,

$$
\begin{aligned}
d I_{w}(t) / d t= & \sum_{i=1}^{N} \varepsilon^{-2} \sum_{j=1}^{N} \int_{\Lambda_{\jmath}(0)} d \mathbf{x}\left\{2\left[\mathbf{x}_{\varepsilon}(\mathbf{x}, t)-\mathbf{z}_{i}(t)\right] \cdot\left[\dot{\mathbf{x}}_{\varepsilon}(\mathbf{x}, t)-\dot{\mathbf{z}}_{i}(t)\right]\right. \\
& \times W\left(\left|\mathbf{\mathbf { x } _ { \varepsilon }}(\mathbf{x}, t)-\mathbf{z}_{i}(t)\right|\right)+\left[\mathbf{x}_{\varepsilon}(\mathbf{x}, t)-\mathbf{z}_{i}\right]^{2} \nabla W\left(\left|\mathbf{x} \mathbf{x}_{\varepsilon}(\mathbf{x}, t)-\mathbf{z}_{i}(t)\right|\right) \\
& \left.\times\left[\dot{\mathbf{x}}_{\varepsilon}(\mathbf{x}, t)-\dot{\mathbf{z}}_{i}(t)\right]\right\} .
\end{aligned}
$$

By Eqs. (1.9), (1.10), (1.16) and the identity

$$
\prod_{k=1, k \neq i}^{N}\left[1-\chi\left(\cdot \mid \Sigma\left(\mathbf{z}_{k}(t), R_{1}\right)\right)\right]+\sum_{k=1, k \neq i}^{N} \chi\left(\cdot \mid \Sigma\left(\mathbf{z}_{k}(t), R_{1}\right)\right)=1
$$

we have

$$
d I_{w}(t) / d t=A+B+D
$$

where

$$
\begin{aligned}
A= & \sum_{i=1}^{N} \varepsilon^{-2} \sum_{j=1}^{N} \int_{A_{j, \varepsilon}(0)} d \mathbf{x} 2\left[\mathbf{x}_{\varepsilon}(\mathbf{x}, t)-\mathbf{z}_{i}(t)\right] \\
& \times\left\{\varepsilon ^ { - 2 } \sum _ { h = 1 } ^ { N } \left[\int_{\Lambda_{h, \varepsilon}(0)} d \mathbf{y K}\left(\mathbf{x}_{\varepsilon}(\mathbf{x}, t)-\mathbf{y}_{\varepsilon}(\mathbf{y}, t)\right)\right.\right. \\
& \left.\times \prod_{k=1, k \neq i}^{N}\left[1-\chi\left(\mathbf{y} \mid \Sigma\left(\mathbf{z}_{k}(t), R_{1}\right)\right]\right] W\left(\left|\mathbf{x}_{\varepsilon}(\mathbf{x}, t)-\mathbf{z}_{i}(t)\right|\right)\right\} \\
B= & \sum_{i=1}^{N} \varepsilon^{-2} \sum_{j=1}^{N} \int_{A_{J, \varepsilon}(0)} d \mathbf{x} 2\left[\mathbf{x}_{\varepsilon}(\mathbf{x}, t)-\mathbf{z}_{i}(t)\right] \\
& \times\left\{\varepsilon ^ { - 2 } \sum _ { h = 1 } ^ { N } \int _ { \Lambda _ { h , \varepsilon } ( 0 ) } \left[d \mathbf { y K } ( \mathbf { x } \mathbf { x } _ { \varepsilon } ( \mathbf { x } , t ) - \mathbf { y } _ { \varepsilon } ( \mathbf { y } , t ) ) \cdot \left[\sum _ { k = 1 , k \neq i } ^ { N } \chi \left(\mathbf{y} \mid \Sigma\left(\mathbf{z}_{k}(t) \mid R_{1}\right)\right.\right.\right.\right. \\
& \left.\left.-\sum_{k=1, k \neq i}^{N} a_{k} \mathbf{K}\left(\mathbf{z}_{i}(t)-\mathbf{z}_{k}(t)\right)\right]\right\} W\left(\left|\mathbf{x}_{\varepsilon}(\mathbf{x}, t)-\mathbf{z}_{i}(t)\right|\right), \\
D= & \sum_{i=1}^{N} \varepsilon^{-2} \sum_{j=1}^{N} \int_{\Lambda_{j, \varepsilon}(0)} d \mathbf{x}\left[\mathbf{x}_{\varepsilon}(\mathbf{x}, t)-\mathbf{z}_{i}(t)\right]^{2} \nabla W\left(\left|\mathbf{x}_{\varepsilon}(\mathbf{x}, t)-\mathbf{z}_{i}(t)\right|\right) \\
& \times\left\{\varepsilon^{-2} \sum_{h=1}^{N} \int_{\Lambda_{h, \varepsilon}(0)} d \mathbf{y K} \mathbf{K}\left(\mathbf{x}(\mathbf{x}, t)-\mathbf{y}_{\varepsilon}(\mathbf{y}, t)\right)-\sum_{h=1, h \neq i}^{N} a_{h} \mathbf{K}\left(\mathbf{z}_{i}(t)-\mathbf{z}_{h}(t)\right)\right\}
\end{aligned}
$$

We study these three terms.

Using the antisymmetry of $\mathbf{K}$ we can write

$$
\begin{aligned}
A= & \sum_{i=1}^{N} \varepsilon^{-4} \sum_{j=1}^{N} \sum_{h=1}^{N} \int_{A_{\jmath}, \varepsilon(0)} d \mathbf{x} \int_{A_{h, \varepsilon}(0)} d \mathbf{y}\left[[ \mathbf { x } _ { \varepsilon } ( \mathbf { x } , t ) - \mathbf { z } _ { i } ( t ) ] \prod _ { k = 1 , k \neq i } ^ { N } \left[1-\chi\left(\mathbf{y} \mid \sum\left(\mathbf{z}_{k}(t) \mid R_{1}\right)\right]\right.\right. \\
& \times W\left(\left|\mathbf{x}_{\varepsilon}(\mathbf{x}, t)-\mathbf{z}_{i}(t)\right|\right)-\left[\mathbf{y}_{\varepsilon}(\mathbf{y}, t)-\mathbf{z}_{i}(t)\right] \prod_{k=1, k \neq i}^{N}\left[1-\chi\left(\mathbf{x} \mid \Sigma\left(\mathbf{z}_{k}(t) \mid R_{1}\right)\right]\right. \\
& \left.\times W\left(\left|\mathbf{y}_{\varepsilon}(\mathbf{y}, t)-\mathbf{z}_{i}(t)\right|\right)\right] \cdot \mathbf{K}\left(\mathbf{x} \mathbf{x}_{\varepsilon}(\mathbf{x}, t)-\mathbf{y}_{\varepsilon}(\mathbf{y}, t)\right) .
\end{aligned}
$$


We note that this term vanishes when both $\left|\mathbf{x}_{\varepsilon}(\mathbf{x}, t)-\mathbf{z}_{i}(t)\right|$ and $\left|\mathbf{y}_{\varepsilon}(\mathbf{y}, t)-\mathbf{z}_{i}(t)\right|$ are less than $R_{1}$. [We use the fact that $\mathbf{K}(\mathbf{x})$ is orthogonal to $\mathbf{x}$.] Moreover, the integrand is bounded. It is not trivial for the singularity of $\mathbf{K}(\mathbf{x}-\mathbf{y})$ as $|\mathbf{x}-\mathbf{y}|$. However, when $\chi$ in (2.11) is $\neq 0$ the points are far and so the integrand is trivially bounded. Otherwise, the integrand is continuous and the term in square brackets vanishes as $|\mathbf{x}-\mathbf{y}|[$ [by Eq. (2.4)] and the identity

$$
\begin{aligned}
\mathbf{x}_{1} W\left(\left|\mathbf{x}_{1}\right|\right)-\mathbf{x}_{2} W\left(\left|\mathbf{x}_{2}\right|\right)= & 1 / 2\left(\mathbf{x}_{1}-\mathbf{x}_{2}\right)\left[W\left(\left|\mathbf{x}_{1}\right|\right)+W\left(\left|\mathbf{x}_{2}\right|\right)\right] \\
& +1 / 2\left(\mathbf{x}_{1}+\mathbf{x}_{2}\right)\left[W\left(\left|\mathbf{x}_{1}\right|\right)-W\left(\left|\mathbf{x}_{2}\right|\right)\right] .
\end{aligned}
$$

We denote by $a_{i}+\mu_{i}(\tau)$ the vorticity contained in $\sum\left(\mathbf{z}_{i}(t) \mid R_{1}\right)$ and

$$
m=\max _{0 \leqq i \leqq N} \sup _{0 \leqq \tau \leqq t}\left|\mu_{i}(\tau)\right|
$$

We have, immediately

$$
|A| \leqq C_{3} m \text {. }
$$

To study $B$, let us denote by $L$ the Lipschitz constant of $\mathbf{K}(\mathbf{x})$ when $|\mathbf{x}|>R_{0} / 2$, $a=\max a_{i}$. Then for $|\mathbf{x}-\mathbf{y}|>R_{0} / 2$ we have

$$
\mathbf{K}(\mathbf{x}-\mathbf{y})=\mathbf{K}\left(\mathbf{z}_{i}-\mathbf{z}_{k}\right)+\mathbf{d} \quad \text { with } \quad|\mathbf{d}|<L\left[\left|\mathbf{x}-\mathbf{z}_{i}\right|+\left|\mathbf{y}-\mathbf{z}_{k}\right|\right] .
$$

Then

$$
\begin{aligned}
& |B| \leqq \sum_{i=1}^{N} \varepsilon^{-2} \sum_{j=1}^{N} \int_{\Lambda_{j, \varepsilon}(0)} d \mathbf{x} 2\left|\mathbf{x}_{\varepsilon}(\mathbf{x}, t)-\mathbf{z}_{i}(t)\right| W\left(\left|\mathbf{x} \varepsilon(\mathbf{x}, t)-\mathbf{z}_{i}(t)\right|\right) \\
& \times\left\{\left[\sum_{h=1}^{N} \varepsilon^{-2} \int_{\Lambda_{h, \varepsilon}(0)} d \mathbf{y} L \sum_{k=1, k \neq i}^{N}\left[\left|\mathbf{x}_{\varepsilon}(\mathbf{x}, t)-\mathbf{z}_{i}(t)\right|+\left|\mathbf{y}_{\varepsilon}(\mathbf{y}, t)-\mathbf{z}_{k}(t)\right|\right]\right.\right. \\
& \times \chi\left(\mathbf{y} \mid \Sigma\left(\mathbf{z}_{k}(t) \mid \mathbf{R}_{1}\right)\right] \\
& \times\left[\sum_{k=1, k \neq i}^{N} \mathbf{K}\left(\mathbf{z}_{i}-\mathbf{z}_{k}\right)\left[a_{k}-\sum_{h=1}^{N} \int_{A_{h, \varepsilon}(0)} d \mathbf{y} \varepsilon^{-2} \chi\left(\mathbf{y} \mid \Sigma\left(\mathbf{z}_{k}(t) \mid R_{1}\right]\right]\right\}\right. \\
& \leqq 2 a N L I_{w}(t)+\sum_{i=1}^{N} \varepsilon^{-4} \sum_{j=1}^{N} \sum_{h=1}^{N} \sum_{k=1, k \neq i}^{N} L \int_{A_{j} c(0)} d \mathbf{x} \int_{\Lambda_{h, \varepsilon}(0)} d \mathbf{y} \\
& \times\left\{2\left|\mathbf{x}_{\varepsilon}(\mathbf{x}, t)-\mathbf{z}_{i}(t)\right|\left|\mathbf{y}_{\varepsilon}(\mathbf{y}, t)-\mathbf{z}_{k}(t)\right| W\left(\left|\mathbf{x}_{\varepsilon}(\mathbf{x}, t)-\mathbf{z}_{i}(t)\right|\right) W\left(\left|\mathbf{y}_{\varepsilon}(\mathbf{y}, t)-\mathbf{z}_{k}(t)\right|\right)\right\}+C_{4} m \\
& \leqq C_{5} I_{w}(t)+C_{6} m \\
& |D| \leqq\left(\varepsilon^{-4}\right) / 2 \sum_{i=1}^{N} \sum_{j=1}^{N} \sum_{h=1}^{N} \int_{A_{j, \varepsilon}(0)} d \mathbf{x} \int_{\Lambda_{h, \varepsilon}(0)} d \mathbf{y} \mid\left\{\left[\mathbf{x} \varepsilon(\mathbf{x}, t)-\mathbf{z}_{i}(t)\right]^{2} \nabla W\left(\left|\mathbf{x} \varepsilon(\mathbf{x}, t)-\mathbf{z}_{i}(t)\right|\right)\right. \\
& \left.\times\left[\mathbf{y}_{\varepsilon}(\mathbf{y}, t)-\mathbf{z}_{i}(t)\right]^{2} \nabla W\left(\left|\mathbf{y}_{\varepsilon}(\mathbf{y}, t)-\mathbf{z}_{i}(t)\right|\right) \cdot \mathbf{K}\left(\mathbf{x}_{\varepsilon}(\mathbf{x}, t)-\mathbf{y}_{\varepsilon}(\mathbf{y}, t)\right)\right\} \mid+C_{7} m .
\end{aligned}
$$

The integrand is bounded as in Eq. (2.11). Hence

$$
|D| \leqq C_{8} m \text {, }
$$

and

$$
\left|d I_{w}(t) / d t\right| \leqq C_{9} I_{w}(t)+C_{10} m
$$


We want now bound $m$ by $I_{w}(t)$. We denote by

$$
I(t)=\sup _{0 \leqq \tau \leqq t} I_{w}(\tau)
$$

Lemma 1. There exists a constant $C_{11}$ such that

$$
m(t) \leqq C_{11} I(t)
$$

Proof of Lemma. We introduce a smooth function $G(r)$

$$
\begin{gathered}
G(r)=\left\{\begin{array}{l}
1 \text { if } 0 \leqq r \leqq R_{1}, \\
a \text { decreasing function if } R_{1} \leqq r \leqq R_{3}, \\
0 \quad \text { if } \quad r \geqq R_{3}, \\
\quad \sup |\nabla G(|\mathbf{x}|)|=C_{12},
\end{array}\right. \\
\left|\nabla G\left(\left|\mathbf{x}_{1}\right|\right)-\nabla G\left(\left|\mathbf{x}_{2}\right|\right)\right| \leqq C_{13}\left|\mathbf{x}_{1}-\mathbf{x}_{2}\right| .
\end{gathered}
$$

We define

$$
m_{i}^{*}=\varepsilon^{-2} \sum_{j=1}^{N} \int_{\Lambda, \varepsilon(0)} G\left(\left|\mathbf{x}_{\varepsilon}(\mathbf{x}, t)-\mathbf{z}_{i}(t)\right|\right) d \mathbf{x} .
$$

Then

$$
\begin{aligned}
& d m_{i}^{*} / d t=\varepsilon^{-2} \sum_{j=1}^{N} \int_{\Lambda_{j, \varepsilon}(0)} \nabla G\left(\left|\mathbf{x}_{\varepsilon}(\mathbf{x}, t)-\mathbf{z}_{j}(t)\right|\right) \cdot\left(\dot{\mathbf{x}}_{\varepsilon}(\mathbf{x}, t)-\dot{\mathbf{z}}_{i}(t)\right) d \mathbf{x} \\
& =\varepsilon^{-4} \sum_{j=1}^{N} \sum_{h=1}^{N} \int_{\Lambda_{,, \varepsilon}(0)} d \mathbf{x} \int_{A_{h, \varepsilon}(0)} d \mathbf{y} \nabla G\left(\left|\mathbf{x}_{\varepsilon}(\mathbf{x}, t)-\mathbf{z}_{i}(t)\right|\right) \\
& \times \mathbf{K}\left(\mathbf{x}_{\varepsilon}(\mathbf{x}, t)-\mathbf{y}_{\varepsilon}(\mathbf{y}, t)\right) \\
& -\varepsilon^{-2} \sum_{j=1}^{N} \sum_{h=1, h \neq i}^{N} \int_{A_{j, \varepsilon}(0)} d \mathbf{x} \nabla G\left(\left|\mathbf{x}_{\varepsilon}(\mathbf{x}, t)-\mathbf{z}_{i}(t)\right|\right) \\
& \times a_{h} \mathbf{K}\left(\mathbf{z}_{i}(t)-\mathbf{z}_{h}(t)\right), \\
& \left|d m_{i}^{*} / d t\right| \leqq 1 / 2 \varepsilon^{-4} \sum_{j=1}^{N} \sum_{h=1}^{N} \int_{\Lambda_{J}, \varepsilon(0)} d \mathbf{x} \int_{\Lambda_{h, \varepsilon}(0)} d y\left[\nabla G\left(\left|\mathbf{x}_{\varepsilon}(\mathbf{x}, t)-\mathbf{z}_{i}(t)\right|\right)\right. \\
& \left.-\nabla G\left(\left|\mathbf{y}_{\varepsilon}(\mathbf{y}, t)-\mathbf{z}_{i}(t)\right|\right)\right] \cdot \mathbf{K}\left(\mathbf{x}_{\varepsilon}(\mathbf{x}, t)-\mathbf{y}_{\varepsilon}(\mathbf{y}, t)\right)+C_{14} m_{i}^{\prime} \\
& \leqq C_{15} m_{i}^{\prime} \text {, }
\end{aligned}
$$

where $m_{i}^{\prime}$ is the vorticity contained in $\Sigma\left(\mathbf{z}_{i}(t) \mid R_{3}\right) / \Sigma\left(\mathbf{z}_{i}(t) \mid R_{1}\right)$.

On the other hand, by the definition of $I(t)$

$$
I(t) \geqq R_{1}^{2} m_{i}^{\prime} / 2 \text {. }
$$

Hence

$$
\left|d m_{i}^{*}(\tau) / d \tau\right| \leqq C_{16} I(t), \quad m_{i}^{*}(0)=a_{i}
$$

that implies

$$
\left|m_{i}^{*}(0)-m_{i}^{*}(t)\right| \leqq C_{16} t I(t)=C_{17} I(t) .
$$


So the vorticity contained in $\Sigma\left(\mathbf{z}_{i}(t) \mid R_{1}\right)$ may differ from $a_{i}$ at most by

$$
C_{17} I(t)+m_{i}^{\prime} \leqq C_{18} I(t) \text {. }
$$

We return to the main theorem. Using Eqs. (2.17) we have proved that

$$
\left|d I_{w}(t) / d t\right| \leqq C_{19} I(t)
$$

that implies

$$
I(t) \leqq I(0) \exp \left(C_{19} t\right)=C_{20} I(0) \leqq C_{21} \varepsilon^{2},
$$

and so

$$
I_{w}(t) \leqq C_{21} \varepsilon^{2} .
$$

By Eqs. (2.19), (2.13) we have

$$
\mu_{i}(t) \leqq C_{22} \varepsilon^{2} .
$$

We have proved, roughly speaking, that the blobs remain concentrated during the motion. The other steps in the proof are, in some sense, easier and they will be only sketched.

We define

$$
\mathbf{M}_{i, \varepsilon, w}(t)=a_{i}^{-1} \sum_{j=1}^{N} \varepsilon^{-2} \int_{A_{j, \varepsilon}(0)} d \mathbf{x} \mathbf{x}_{\varepsilon}(\mathbf{x}, t) W\left(\left|\mathbf{x}_{\varepsilon}(\mathbf{x}, t)-\mathbf{Z}_{i}(t)\right|\right) .
$$

We have

$$
\begin{aligned}
& d \mathbf{M}_{i, \varepsilon, w}(t) / d t=a_{i}^{-1} \sum_{j=1}^{N} \varepsilon^{-2} \int_{\Lambda_{j, \varepsilon}(0)} d \mathbf{x}\left\{\dot{\mathbf{x}}_{\varepsilon}(\mathbf{x}, t) W\left(\left|\mathbf{x}_{\varepsilon}(\mathbf{x}, t)-\mathbf{z}_{i}(t)\right|\right)\right. \\
& \left.+\mathbf{x}_{\varepsilon}(\mathbf{x}, t) \boldsymbol{\nabla} W\left(\left|\mathbf{x}_{\varepsilon}(\mathbf{x}, t)-\mathbf{z}_{i}(t)\right|\right) \cdot\left(\dot{\mathbf{x}}_{\varepsilon}(\mathbf{x}, t)-\dot{\mathbf{z}}_{i}(t)\right)\right\}, \\
& \left|d\left[\mathbf{M}_{i, \varepsilon, w}(t)-\mathbf{Z}_{i}(t)\right] / d t\right| \leqq \mid a_{i}^{-1} \varepsilon^{-4} \sum_{j=1}^{N} \sum_{h=1}^{N}\left\{\int_{A_{j, \varepsilon}(0)} \int_{A_{h, \varepsilon}(0)} d \mathbf{x} d \mathbf{y}\right. \\
& \left.\times W\left(\mid \mathbf{x}_{\varepsilon}(\mathbf{x}, t)\right)-\mathbf{z}_{i}(t) \mid\right)\left[\prod_{k=1, k \neq i}^{N}\left(1-\chi\left(\mathbf{y} \mid \Sigma\left(\mathbf{z}_{k}(t) \mid R_{1}\right)\right)\right)\right. \\
& \left.\left.+\sum_{k=1, k \neq i}^{N} \chi\left(\mathbf{y} \mid \Sigma\left(\mathbf{z}_{k}(t) \mid R_{1}\right)\right)\right] \mathbf{K}\left(\mathbf{x}_{\varepsilon}(\mathbf{x}, t)-\mathbf{y}_{\varepsilon}(\mathbf{y}, t)\right)\right\} \\
& -\sum_{k=1, k \neq i}^{N} a_{k} \mathbf{K}\left(\mathbf{z}_{i}(t)-\mathbf{z}_{k}(t)\right) \mid+C m_{23} \\
& \leqq C_{24} m+\mid\left\{a_{i}^{-1} \varepsilon^{-4} \sum_{j=1}^{N} \sum_{h=1}^{N} \sum_{k=1, k \neq i}^{N} \int_{\Lambda_{j, \varepsilon}(0)} \int_{\Lambda_{h, \varepsilon}(0)} d \mathbf{x} d \mathbf{y}\right. \\
& \times L W\left(\left|\mathbf{x}_{\varepsilon}(\mathbf{x}, t)-\mathbf{z}_{i}(t)\right|\right) \chi\left(\mathbf{y} \mid \Sigma\left(\mathbf{z}_{k}(t) \mid R_{1}\right)\right. \\
& \times\left\{\left[\left(\mathbf{x}_{\varepsilon}(\mathbf{x}, t)-\mathbf{z}_{i}(t)\right]+\left[\mathbf{y}_{\varepsilon}(\mathbf{y}, t)-\mathbf{z}_{k}(t)\right]\right\}\right. \\
& +W\left(\left|\mathbf{x}_{\varepsilon}(\mathbf{x}, t)-\mathbf{z}_{i}(t)\right|\right) \chi\left(\mathbf{y} \mid \sum\left(\mathbf{z}_{k}(t) \mid R_{1}\right) \mathbf{K}\left(\mathbf{z}_{i}(t)-\mathbf{z}_{k}(t)\right)\right\} \\
& -\sum_{k=1, k \neq i}^{N} a_{k} \mathbf{K}\left(\mathbf{z}_{i}(t)-\mathbf{z}_{k}(t)\right)
\end{aligned}
$$




$$
\begin{aligned}
\leqq & C_{25} m+L a_{i}^{-1} \varepsilon^{-4} \sum_{j=1}^{N} \sum_{h=1}^{N} \sum_{k=1, k \neq i}^{N} \int_{A_{j, \varepsilon}(0)} \int_{A_{h, \varepsilon}(0)} d \mathbf{x} d \mathbf{y} \\
& \times\left\{W\left(\left|\mathbf{x}_{\varepsilon}(\mathbf{x}, t)-\mathbf{z}_{i}(t)\right|\right)\left|\mathbf{x}_{\varepsilon}(\mathbf{x}, t)-\mathbf{z}_{i}(t)\right|\right. \\
& \left.+W\left(\left|\mathbf{y}_{\varepsilon}(\mathbf{y}, t)-\mathbf{z}_{k}(t)\right|\right)\left|\mathbf{y}_{\varepsilon}(\mathbf{y}, t)-\mathbf{z}_{k}(t)\right|\right\} \\
\leqq & C_{26} m+C_{27} \varepsilon^{-2} \sum_{j=1}^{N} \sum_{k=1}^{N} \int_{A_{1, \varepsilon}(0)} d \mathbf{x} \\
& \times\left|\mathbf{x}_{\varepsilon}(\mathbf{x}, t)-\mathbf{z}_{k}(t)\right| W\left(\left|\mathbf{x}_{\varepsilon}(\mathbf{x}, t)-\mathbf{z}_{k}(t)\right|\right) \\
\leqq & C_{26} m+C_{28}\left[\varepsilon^{-2} \sum_{j=1}^{N} \sum_{k=1}^{N} \int_{A_{J, k}(0)} d \mathbf{x}\right. \\
& \left.\times\left|\mathbf{x}_{\varepsilon}(\mathbf{x}, t)-\mathbf{z}_{k}(t)\right|^{2} W\left(\left|\mathbf{x}_{\varepsilon}(\mathbf{x}, t)-\mathbf{z}_{k}(t)\right|\right)^{2}\right]^{1 / 2} \\
\leqq & C_{26} m+C_{29} I(t)^{1 / 2} \leqq C_{30^{\varepsilon}}-\underset{\varepsilon \rightarrow 0}{ }, 0
\end{aligned}
$$

by the previous estimates and the Cauchy-Schwarz inequality.

The initial data are similar and so we have proved that

$$
\mathbf{M}_{i, \varepsilon, w}(t) \underset{\varepsilon \rightarrow 0}{\underset{1}{-} \rightarrow \mathbf{Z}_{i}}(t) .
$$

Now we compare $\mathbf{M}_{i, \varepsilon}(t)$ with $\mathbf{M}_{i, \varepsilon, w}(t)$. They differ by the vorticity inivially in $\Sigma\left(\mathbf{z}_{i} \mid R_{1}\right)$ that has gone out and by the vorticity entered in $\Sigma\left(\mathbf{z}_{i}(t) \mid R_{2}\right)$. We realize that each two are bounded by $C \varepsilon^{2}$. In fact, in the region out of $\Sigma\left(\mathbf{z}_{i}(\tau) \mid R_{2}\right)$ for any $i$ and $\tau$, $0 \leqq \tau \leqq t$, the vorticity is bounded by $C \varepsilon^{2}$ and consequently the field is bounded by a constant. (It is easy to observe that a blob of vorticity $\varepsilon^{2}$ and intensity $\varepsilon^{-2}$ produces a velocity field

$$
\left.\leqq \varepsilon^{-2}(2 \pi)^{-1} \int_{|\mathbf{x}| \leqq r}|\mathbf{x}|^{-1} d \mathbf{x}, \text { where } r=\varepsilon^{2}(\pi)^{-1 / 2} \cdot\right)
$$

Hence the vorticity flux cannot be too large. Moreover, the fluid remains in a bounded region (for finite $t$ ). So the contribution to $\mathbf{M}_{i, \varepsilon}(t)$ of the "particles" far away from $\boldsymbol{z}_{i}(t)$ cannot be too large.

In conclusion we have proved

$$
\left|\mathbf{M}_{i, \varepsilon}(t)-\mathbf{M}_{i, \varepsilon, w}(t)\right| \leqq C \varepsilon_{\varepsilon \rightarrow 0}^{2} \underset{\varepsilon \rightarrow 0}{\longrightarrow \rightarrow} 0,
$$

and this achieves the proof.

\section{Remarks}

In the previous section we have also proved the weak convergence of the measure $\omega_{\varepsilon}(\mathbf{x}, t)$ :

Theorem 3.1. Let $f: R^{2} \rightarrow R$ be any continuous function. Then for all $t$,

$$
\lim _{\varepsilon \rightarrow 0} \int f(\mathbf{x}) \omega_{\varepsilon}(\mathbf{x}, t) d \mathbf{x}=\sum_{i=1}^{N} a_{i} f\left(\mathbf{z}_{i}(t)\right) .
$$


Now we want to show a simple case in which the filaments of vorticity remain close to the center of vorticity when $\varepsilon \rightarrow 0$. We consider a (noncircular) blob of vorticity moving in absence of external fields. The center of vorticity remains fixed, but thin filaments evolve in a complicated way and, for long time, may go far away. We show that the limit $\varepsilon \rightarrow 0$ prevents this effect in spite of the fact that the vorticity density becomes infinite.

Theorem 3.2. Let

$$
\omega(\mathbf{x}, 0)=\varepsilon^{-2} \chi\left(\mathbf{x} \mid \Lambda_{\varepsilon}(0)\right)
$$

with

$$
\operatorname{meas} \Lambda_{\varepsilon}(0)=\varepsilon^{2}, \quad \Lambda_{\varepsilon}(0) \subset \Sigma(0 \mid \varepsilon),
$$

and let

$$
\omega(\mathbf{x}, t)=\varepsilon^{-2} \chi\left(\mathbf{x} \mid \Lambda_{\varepsilon}(t)\right),
$$

the evolution of $\omega(\mathbf{x}, 0)$ via the Euler equations. Then for any $t$ and any $d$

$$
\left[\lim _{\varepsilon \rightarrow 0} \Lambda_{\varepsilon}(t)\right] \subset \Sigma(0, d) .
$$

Proof. We sketch the proof. It is enough to show that the radial component of the velocity field vanishes when $\varepsilon \rightarrow 0$ for any fixed $t$ and $|\mathbf{x}|$. We consider

$$
\begin{gathered}
\left.\mu\left(R_{1}\right)=1-\varepsilon^{-2} \int_{A_{\varepsilon}(0)} d \mathbf{x} W\left(\mid \mathbf{x}_{\varepsilon}(\mathbf{x}, t)\right)\right), \\
d \mu\left(R_{1}\right) / d t=-\varepsilon^{-2} \int_{\Lambda_{\varepsilon}(0)} d \mathbf{x} \nabla W \cdot \dot{\mathbf{x}} \\
=-\varepsilon^{-4} \int_{\Lambda_{\varepsilon}(0)} d \mathbf{x}\left\{\nabla W\left(\left|\mathbf{x}_{\varepsilon}(\mathbf{x}, t)\right|\right) \cdot \int_{\Lambda_{\varepsilon}(0)} d \mathbf{y K}\left(\mathbf{x}_{\varepsilon}(\mathbf{x}, t)-\mathbf{y}_{\varepsilon}(\mathbf{y}, t)\right)\right\} \\
=-\varepsilon^{-4} 1 / 2 \int_{A_{\varepsilon}(0)} \int_{\Lambda_{\varepsilon}(0)}\left\{d \mathbf{x} d \mathbf{y}\left[\mathbf{\nabla} W\left(\left|\mathbf{x}_{\varepsilon}(\mathbf{x}, t)\right|\right)-\nabla W\left(\left|\mathbf{y}_{\varepsilon}(\mathbf{y}, t)\right|\right)\right]\right. \\
\left.\quad \times \mathbf{K}\left(\mathbf{x}_{\varepsilon}(\mathbf{x}, t)-\mathbf{y}_{\varepsilon}(\mathbf{y}, t)\right)\right\} .
\end{gathered}
$$

The integrand is bounded by a constant proportional to $C_{2}$.

From now on, we choose $R_{2}=3 / 2 R_{1}, C_{2}=\sigma R_{1}^{-2}$, where $\sigma$ is independent of $R_{1}$. We consider $\gamma$ such that $\varepsilon \ll \gamma \ll R_{1}$ and we divide the integral in $\mathbf{y}$ in Eq. (3.3) into two parts: $|\mathbf{y}| \leqq \gamma$ and $|\mathbf{y}|>\gamma$. We denote by $m(R)$ the vorticity external to $\Sigma(0, R)$. Then, the first contribution is proportional to $m\left(R_{1}\right) \gamma R_{1}^{-2}$. (In fact, for $\gamma \ll R_{1} \nabla W$ and $\mathbf{K}$ are almost orthogonal.) The second contribution is proportional to $m(\gamma) m\left(R_{1}\right) \sigma R_{1}^{-2}$. Hence

$$
\left|d \mu\left(R_{1}\right) / d t\right| \leqq C m\left(R_{1}\right) \gamma R_{1}^{-2}+C m(\gamma) m\left(R_{1}\right) R_{1}^{-2} .
$$

By definition, we have

$$
m(R) \leqq I /(R)^{2},
$$

where

$$
I=\varepsilon^{-2} \int_{\Lambda_{\varepsilon}(0)} d \mathbf{x}\left|\mathbf{x}_{\varepsilon}(\mathbf{x}, t)\right|^{2},
$$


which is constant and proportional to $\varepsilon^{2}$. Then

$$
\left|d \mu\left(R_{1}\right) / d t\right| \leqq \varepsilon^{2}\left[C \gamma R_{1}^{-4}+C \varepsilon^{2} \gamma^{-2} R_{1}^{-4}\right] \rightarrow 0 \quad \text { as } \varepsilon^{\beta}, \quad \beta>2,
$$

if we choose $\gamma \approx \varepsilon^{1 / 2}, R_{1} \approx \gamma^{1 / 5}$. Then

$$
\mu\left(R_{1}\right) \rightarrow 0 \quad \text { as } \varepsilon^{\beta}, \quad \beta>2,
$$

and

$$
m\left(R_{2}\right) \leqq \mu\left(R_{1}\right) \rightarrow 0
$$

so that

$$
m\left(R_{2}\right) \rightarrow 0 \quad \text { as } \varepsilon^{\beta}, \quad \beta>2,
$$

and the velocity field produced by this vorticity vanishes as $\varepsilon^{-2+\beta}$ [Eq. (2.38)]. Finally, we consider an $\mathbf{x}$ such that $|\mathbf{x}|=R_{4} \gg R_{2}$; in this point both the velocity field produced by the vorticity out of $\Sigma\left(0 \mid R_{2}\right)$ and the radial component of the field generated by the vorticity in $\Sigma\left(0 \mid R_{2}\right)$ vanish.

We note that this proof fails in the presence of external forces.

In the presence of boundaries the existence of a global solution for the vortex Eq. (1.16) may fail. In fact, an eventual collision between the vortices and the boundary is not forbidden by the conservation of energy. Nevertheless, initial data producing this collapse should be exceptional. This has been proved rigorously for a circular domain [2] and it can be conjectured to be true in general. For all "good" initial conditions the technique of the present paper can be used and the result remains valid.

The proof of Sect. 2 fails when the vortices have different signs. In fact, in this case we are not able to control the growth in time of $I_{w}$; moreover, $I_{w}$ is not a good Liapunov function of the problem.

Finally, we can study the stability of the measure with respect to a small viscosity. Let $\tilde{\omega}_{\varepsilon, v}(\mathbf{x}, t)$ be the evolution of the initial data (1.13), (1.14) via the Navier-Stokes equations:

$$
\partial_{t} \tilde{\omega}_{\varepsilon, v}(\mathbf{x}, t)+(\tilde{\mathbf{u}} \cdot \nabla) \tilde{\omega}_{\varepsilon, v}(\mathbf{x}, t)=v \Delta \tilde{\omega}_{\varepsilon, v}(\mathbf{x}, t), \quad \mathbf{x} \in R^{2} .
$$

It is well known that the solution of the Navier-Stokes. equations for bounded initial data converge to the solutions of the Euler equations when $v \rightarrow 0$. In our case also, it is reasonable to prove, using the ideas of Sect. 2, that

$$
\tilde{\omega}_{\varepsilon, v}(\mathbf{x}, t) \underset{\substack{\varepsilon \rightarrow 0 \\ v \rightarrow 0}}{\stackrel{\text { weak }}{\longrightarrow}} \sum_{i=1}^{N} a_{i} \delta\left(\mathbf{z}_{i}(t)\right) .
$$

[ $\delta(\mathbf{x})$ denotes the Dirac measure concentrated in $\mathbf{x}$.

Note that for singular initial data the uniqueness of the solution of the NavierStokes equations is only proved for large enough viscosity [6].

Acknowledgements. It is a pleasure to thank the "Centre de Physique Théorique, CNRS Luminy, Marseille," where part of this research has been performed, for its warm hospitality. 


\section{References}

1. Helmholtz, H.: On the integrals of the hydrodynamical equations which express vortex motion. Phys. Mag. 33, 485 (1867)

Kirchhoff, G.: Vorlesungen über Math. Phys. Leipzig: Teubner 1876

Poincaré, H.: Theories des tourbillons. Paris: George Carré 1893

Kelvin, L.: Mathematical and physical papers. Cambridge: Cambridge University Press 1910

2. Marchioro, C., Pulvirenti, M.: Vortex methods in two-dimensional fluid mechanics. Lecture Notes in Physics, Vol. 203. Berlin, Heidelberg, New York: Springer 1984

3. Marchioro, C., Pulvirenti, M.: Euler evolution for singular initial data and vortex theory. Commun. Math. Phys. 91, 563 (1983)

4. Turkington, B.: On the evolution of a concentrated vortex in an ideal fluid. Northwestern University, Evanston 1984

5. Marchioro, C., Pagani, E.: Evolution of two concentrated vortices in a two-dimensional bounded domain. Math. Methods Appl. Sci. 8, 328 (1986)

6. Benfatto, G., Esposito, R., Pulvirenti, M.: Planar Navier-Stokes flow for singular initial data. Nonlinear Anal., Theory Methods Appl. 9, 533 (1985)

Communicated by J. L. Lebowitz

Received January 6, 1987; in revised form October 8, 1987 
